\title{
Distribuição espacial de populações de triatomíneos (Hemiptera: Reduviidae) em palmeiras da espécie Mauritia flexuosa no Distrito Federal, Brasil
}

\author{
Spatial distribution of Triatominae populations (Hemiptera: Reduviidae) in \\ Mauritia flexuosa palm trees in Federal District of Brazil
}

\author{
Rodrigo Gurgel-Gonçalves ${ }^{1}$, Marco Antônio Duarte ${ }^{1}$, Eduardo Dias Ramalho ${ }^{1}$, \\ Alexandre Ramlo Torre Palma ${ }^{2}$, Christine Agueda Romaña ${ }^{3,4}$ \\ e César Augusto Cuba-Cuba ${ }^{1}$
}

\begin{abstract}
RESUM0
Para determinar eanalisara distribuição espacial de populaçõessilvestres de triatomíneos no Distrito Federal, Brasil, foram amostradas 150 palmeiras da espécie Mauritia flexuosa em seis veredas de diferentes paisagens ( silvestre, rural e periurbana) na estação chuvosa. Os triatomíneos foram identificados morfologicamente, separados por sexo e estadio ninfal e examinados para verificar infecção por Trypanosoma cruzi e Trypanosoma rangeli. Vinte e oito (18,6\%) palmeiras estavam infestadas por Rhodnius neglectus e 14 (9,5\%) por Psammolestes tertius. Afreqüência dessas espécies em palmeiras com e sem ninhos foi significativamente diferente, sendo superior nas palmeiras com ninhos de aves e mamíferos na copa. O maior número médio de insetos/palmeira foi observado nas áreas rurais, com estimativas de até 838 insetos/hectare. A composição etária das espécies apresentou um padrão diferente, Rhodnius neglectus com predomínio de ninfas e Psammolestes tertius com predomínio de adultos, sendo que muitos ovos de Rhodnius neglectus foram coletados indicando um evento reprodutivo em fevereiro de 2003. Entre os ninhos encontrados nas palmeiras, o do pássaro Phacellodomus ruber (Furnariidae) apresentou as maiores abundâncias de triatomíneos, ocorrendo em $42 \%$ das palmeiras. A abundância relativa de Rhodnius neglectus e Psammolestes tertius foi maior em áreas rurais que possuíram maior número de ninhos nas palmeiras e menor densidade de palmeiras por hectare. Nenhum dos 96 triatomíneos examinados estava infectado por Trypanosoma cruzi ou Trypanosoma rangeli, apesar de encontrarmos infecção de Rhodnius neglectus por esses parasitas em estudos anteriores.
\end{abstract}

Palavras-chaves: Rhodnius neglectus. Psammolestes tertius. Mauritia flexuosa. Distrito Federal. Brasil.

\begin{abstract}
To determine and analyze the distribution of Triatominae sylvatic populations in the Federal District of Brazil, 150 Mauritia flexuosa palm trees were sampled in six veredas of different landscapes (sylvatic, rural and peri-urban) in the rainy season. Triatomines were morphologically identified and grouped by sex and nymphal stage and were examined to verify infection by Trypanosoma cruzi and Trypanosoma rangeli. Twenty eight (18.6\%) palm trees were infested by Rhodnius neglectus and fourteen (9.5\%) by Psammolestes tertius. The frequency of Triatominae in palm trees with and without nests was significantly different being higher in palm trees with bird and mammal nests in the crown. The higher average number of insects/palm tree was observed in rural areas with estimates of up to 838 insects/hectare. The species age makeup presented a different pattern, with nymphs predominant for Rhodnius neglectus and adults predominant for Psammolestes tertius. Also, many Rhodnius neglectus eggs were collected, which indicates a reproductive event in February 2003. Among thenests found in palm trees, that of the Phacellodomus ruber (Furnariidae) bird had the greatest abundance of Triatominae, occurring on $42 \%$ of palm trees. The relative abundance of Rhodnius neglectus and Psammolestes tertius was greater in rural areas which contained higher number of nests in palm trees and lesser density of palms per hectare. None of the 96 triatomines examined were infected by Trypanosoma cruzi or Trypanosoma rangeli, despite our finding of Rhodnius neglectus infection by those parasites in former studies.
\end{abstract}

Key-words: Rhodnius neglectus. Psammolestes tertius. Mauritia flexuosa. Federal District of Brazil.

1. Laboratório de Parasitologia Médica e Biologia de Vetores da Área de Patologia da Faculdade de Medicina da Universidade de Brasília, Brasília DF, Brasil. 2. Universidade Católica de Brasília, Brasília DF, Brasil. 3. Centro de Desenvolvimento Sustentável da Universidade de Brasília, Brasília DF, Brasil. 4. UR016, Institut de Recherches pour le Développement, França.

Pesquisa parcialmente financiada pelo CNPq e FINATEC.

Endereço para correspondência: Prof. César Augusto Cuba-Cuba. LPM/BV/AP/FM/UnB, Campus Universitário Darcy Ribeiro, Asa Norte, 70910-900 Brasilia, DF, Brasil.

Fax: 5561273.3907.

e-mail: cuba@unb.br

Recebido para publicação em 9/1/2004

Aceito em 19/4/2004 
0 conhecimento disponível sobre a ocorrência de triatomíneos (Hemiptera: Reduviidae) no Distrito Federal (DF) foi resultado do trabalho de controle das infestações domiciliares e peridomiciliares, realizado pela Fundação Nacional de Saúde/ Ministério da Saúde, Brasil. Avigilância entomológica foi iniciada no início da década de 80, a partir da instalação de uma rede de postos de informações de triatomíneos ( PITs) funcionando com auxílio da população e tendo como objetivos informar, divulgar e atender as denúncias, encaminhando os insetos para exames laboratoriais e realizando conseqüentes borrifações nas unidades domiciliares positivas ${ }^{1}$. Neste trabalho, espécies de triatomíneos como Panstrongylus megistus ( Burmeister, 1835), Triatoma pseudomaculata (Corrêa e Espínola, 1964), Triatoma sordida ( Stal, 1859) e Rhodnius neglectus ( Lent, 1954) foram listadas ${ }^{37}{ }^{38}$. Entretanto, a origem silvestre dessas populações e o potencial papel dessas espécies como vetores de Trypanosoma cruzi e/ou T. rangeli não foram estabelecidos nessa área.

Vários estudos têm salientado a importância de palmeiras como ecótopos naturais de triatomíneos, principalmente de espécies do gênero Rhodnius, importantes vetores da doença de Chagas nas Américas ${ }^{4}{ }^{32}$ cuja distribuição em ambiente silvestre na América Latina coincide fortemente com a distribuição das palmeiras ${ }^{1016}$. Grande controvérsia existe sobre a posição taxonômica de algumas espécies de Rhodnius, que pertencem ao complexo prolixus: R. prolixus (Stal, 1879), R. robustus (Larrousse, 1927), R. nasutus (Stal, 1859) e R. neglectus, que ocorrem no Brasil e cuja determinação exige muitos cuidados ${ }^{2}{ }^{34}$. Dujardin et al $^{12}$ manifestam sua preocupação em relação a qual espécie de Rhodnius está invadindo as casas no Brasil a partir do ambiente silvestre.

Romaña et $\mathrm{a}^{32}$ sugerem que as palmeiras podem ser consideradas como indicadores ecológicos de áreas de risco da doença de Chagas, sendo úteis na identificação da presença de vetores e hospedeiros dos ciclos silvestres de transmissão do T. cruzi. Estudos em diferentes áreas do Brasil têm mostrado altas taxas de infestação de Triatominae, principalmente do gênero Rhodnius em palmeiras ${ }^{3411242527283940}$. Aampla distribuição das palmeiras, sua variabilidade morfológica, que favorece a nidificação de fauna variada e triatomíneos, e seus variados usos pelo homem contribuem para valorizar a importância desse grupo no contexto da Tripanossomíase Americana. № DF, existem oito gêneros e dezesseis espécies de palmeiras, entre elas Mauritia flexuosa L.f. buritis, Acrocomia aculeata (Jacq) Lodd. ex. Mart. macaúbas e Attalea phalerata Mart. ex Spreng. babaçus ${ }^{21}$ que poderiam abrigar triatomíneos silvestres.

Embora no DF não tenha sido documentada transmissão humana autóctone do T. cruzi em ambiente domiciliar ou silvestre, há um número estimado de soropositivos de 71.736 segundo inquérito sorológico ${ }^{38}$. Comparado com outros Estados este número é alto, mesmo não sendo uma área endêmica. Nesse sentido, a possibilidade de invasão e/ou domiciliação de espécies de triatomíneos presentes em ecótopos silvestres podem significar risco de instalação de ciclos peridomiciliares e domiciliares pelo T. cruzi na área. Este estudo propõe-se a determinar e avaliar a distribuição espacial de triatomíneos silvestres associados à palmeira $\mathrm{M}$. flexuosa no DF, contribuindo para o melhor conhecimento sobre a ecologia e distribuição de triatomíneos no Brasil.

\section{MATERIAL E MÉTODOS}

0 Distrito Federal está localizado entre os paralelos de 15030'

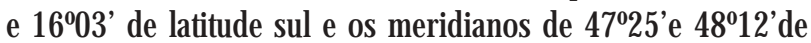
longitude oeste, na região Centro-Oeste, ocupando 0 centro do Brasil e 0 centro-leste do Estado de Goiás em uma das áreas mais elevadas da região, o Planalto Central. As características ambientais da região estudada são: (i) $1000 \mathrm{~m}$ de altitude média, (ii) precipitação média anual de $1545 \mathrm{~mm}$, (iii) temperatura media anual de $21,1^{\circ} \mathrm{C}$, (iv) marcada sazonalidade, com uma estação seca de maio a setembro ( meses com precipitação menor que $100 \mathrm{~mm}$ ) e uma estação chuvosa durante 0 resto do ano.

A pesquisa de triatomíneos foi realizada em formações savânicas chamadas veredas, campos úmidos permanentes, colonizados por populações de palmeiras da espécie M. flexuosa e algumas espécies arbustivas ${ }^{30}$. Essa fitofisionomia tem importante papel na manutenção dos recursos hídricos e, atualmente, é considerada como Area de Proteção Permanente (APP) segundo o Instituto Brasileiro do Meio Ambiente e Recursos Naturais Năo Renováveis (IBAMA).

As 150 palmeiras amostradas estavam localizadas em veredas com diferentes graus de perturbação ambiental, sendo classificadas em três paisagens, de acordo com a área de contato presente na periferia da vereda. Na paisagem silvestre a área de contato era a vegetação nativa ( matas galerias e cerrado strictu sensu); na paisagem rural, plantações e pastos e na paisagem periurbana, conjuntos habitacionais. Foram escolhidas duas áreas em cada paisagem, totalizando seis diferentes áreas de amostragem no DF (Tabela 1). Em cada uma das áreas foi estimada a densidade das palmeiras pelo método proposto por Greig-Smith ${ }^{17}$, baseado nas distâncias das palmeiras mais próximas aos pontos de amostragem.

A amostragem de triatomíneos nas veredas foi feita utilizando transectos de $260 \mathrm{~m}$ de comprimento, divididos em 13 pontos regularmente espaçados ( $20 \mathrm{~m})$, com exceção do transecto da vereda do IBGE, que foi dividido em seis pontos com espaçamento de $40 \mathrm{~m}$ devido a menor densidade de palmeiras na área. As coletas foram feitas durante 0 mês de fevereiro de 2003 (estação chuvosa). As palmeiras mais próximas de cada ponto foram amostradas, totalizando vinte cinco palmeiras por transecto, resultando um mesmo esforço amostral por área de estudo. Os parâmetros morfométricos medidos das palmeiras foram altura da estipe, diâmetro da copa e número de folhas. As palmeiras foram escaladas e 0 material presente na copa ( matéria orgânica, folhas e ninhos abandonados) foi coletado em sacos plásticos ${ }^{19}$ e processado no laboratório, onde os triatomíneos foram separados por 
Tabela 1 - Paisagem, localização e coordenadas geográficas dasáreas de amostragem de populações de triatomíneos em Veredas no Distrito Federal, Brasil, 2003.

\begin{tabular}{|c|c|c|}
\hline Paisagem & Local & Coordenadas geográficas* \\
\hline Silvestre & Estação Ecológica Águas Emendadas, Córrego Vereda Grande. & $15^{0} 34^{\prime} 27^{\prime \prime} \mathrm{S}, 47{ }^{\circ} 36^{\prime} 28^{\prime \prime} \mathrm{W}$ \\
\hline Silvestre & Reserva Ecológica do IBGE, Córrego Taquara. & $15^{0} 55^{\prime} 54^{\prime \prime} \mathrm{S}, 47054^{\prime} 02^{\prime \prime} \mathrm{W}$ \\
\hline Rural & Colônia Agrícola Alphaville, divisa DF/Goiás & $16004^{\prime} 05^{\prime \prime} \mathrm{S}, 47032^{\prime} 39^{\prime \prime} \mathrm{W}$ \\
\hline Rural & Colônia Agrícola Rajadinha, Fazenda Changrilá & $15 \div 46^{\prime} 14^{\prime \prime} \mathrm{S}, 47038^{\prime} 58^{\prime \prime} \mathrm{W}$ \\
\hline Periurbana & Setor de Mansões Park Way Quadra 16 & 15054'12"S, 47056'51"W \\
\hline Periurbana & Samambaia, Fazenda Mocambo & $15 \div 54^{\prime} 12^{\prime \prime} \mathrm{S}, 48 \div 09^{\prime} 02^{\prime \prime} \mathrm{W}$ \\
\hline
\end{tabular}

* Obtidas usando GPS = Geographical Position System

sexo e estádio ninfal, contabilizados e identificados morfologicamente de acordo com chaves descritas ${ }^{20}$. As fezes dos triatomíneos coletados foram examinadas seguindo a técnica empregada para infecção pelo T. cruzi $^{9} \mathrm{e}$ exame de hemolinfa e glândulas salivares dos insetos para a pesquisa de T. rangeli ${ }^{8}$.

Os parâmetros usados na análise da distribuição espacial das populações de triatomíneos nas palmeiras foram a freqüência (número de palmeiras infestadas/número total de palmeiras amostradas na área) e a abundância (número médio de insetos por palmeira infestada na área e abundância relativa de insetos entre as áreas) de acordo com a paisagem (silvestre, rural e periurbana). Foram feitas também estimativas de densidades de triatomíneos nas palmeiras a partir do número médio de indivíduos por palmeira infestada, multiplicado pela densidade de palmeiras e pela percentagem de palmeiras infestadas nas áreas ${ }^{22}$.

As características das palmeiras foram relacionadas com abundância das populações de triatomíneos, por correlação de Spearman ${ }^{41}$ e testes qui-quadrado foram usados para comparar as freqüências de triatomíneos nas áreas estudadas e as freqüências de triatomíneos em palmeiras com e sem ninhos nas bainhas foliares.

\section{RESULTAD0S}

Frequência de palmeiras infestadas por triatomíneos. Entre as 150 palmeiras amostradas, $28(18,6 \%)$ estavam infestadas por R. neglectus e 14 (9,5\%) por Psammolestes tertius. As frequiências dessas espécies foram superiores nas palmeiras com ninhos de aves e mamíferos ( $n=62) \quad(45,1 \%$ e $22,5 \%$ respectivamente). Em nenhuma palmeira, foi observada a infestação exclusiva por P. tertius, ou seja, esta espécie só ocorreu em palmeiras que também estavam infestadas por R. neglectus. Por outro lado, infestações exclusivas por R. neglectus foram observadas em $50 \%$ das palmeiras infestadas.

Houve diferença significativa entre a freqüência de palmeiras infestadas em cada área por $R$. neglectus $\left(X^{2}=15,6\right.$; $\mathrm{p}<0,01 ; \mathrm{n}=28)$ e P. tertius $\left(\mathrm{X}^{2}=11,9 ; \mathrm{p}<0,05 ; \mathrm{n}=14\right)$. A vereda da Samambaia foi a única em que não foram detectadas palmeiras infestadas, nas outras os índices de infestação nas palmeiras variaram de 12 a $40 \%$ para $R$ neglectus e 4 a 24\% para P. tertius ( Tabela 2).

Abundância dos triatomíneos nas palmeiras, estrutura etária e infecção natural por tripanossomatídeos. Foram coletados 332 indivíduos sendo 189 (57\%) R. neglectus e 143 (43\%) P. tertius. Nenhum dos 96 triatomíneos examinados estava infectados por T. cruzi ou T. rangeli. Considerando o número total de indivíduos de ambas as espécies em todas as palmeiras infestadas, 0 número médio de insetos por palmeira foi de $11,9 \pm 11,6$ sendo de $6,8 \pm 5,7$ para $R$. neglectus e $10,2 \pm 7,1$ para P. tertius. 0 número médio de insetos por palmeira em cada área variou de 3,0 a 9,8 para $R$ neglectus e de 3 a 16 para P. tertius e as estimativas de densidade de insetos nas áreas variaram de 57 a 378 indivíduos/ha e 19 a 460 indivíduos/ha respectivamente (Tabela 2), sendo maiores em Alphaville e Rajadinha ( paisagem rural), seguido de Águas Emendadas e IBGE (paisagem silvestre). A correlação entre a abundância de $R$. neglectus e $P$. tertius nas cinco áreas infestadas foi significativa para $p<0,05\left(R_{s}=0,88\right.$; $\mathrm{p}=0,018$ ) sugerindo que a abundância de ambas as espécies nas áreas é influenciada pelos mesmos fatores.

A composição etária das populações R. neglectus e P. tertius em M. flexuosa mostrou um padrão diferente. Nas

Tabela 2 - Índices deinfestação de Rhodnius neglectus e Psammolestes tertius em palmeiras da espécie Mauritia flexuosa em diferentes paisagens eáreas, com um mesmo esforço de amostragem (25 palmeiras por área) no Distrito Federal, Brasil, 2003.

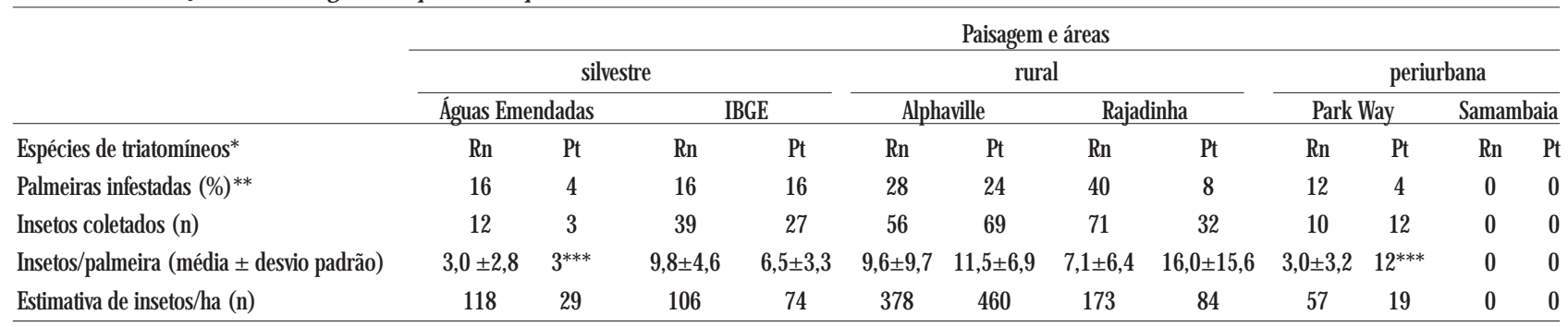

* $\mathrm{Rn}=$ Rhodnius neglectus, $\mathrm{Pt}=$ Psammolestes tertius

$* * X^{2}=15,6 ; p<0,01$ para $R$. neglectus e $X^{2}=11,9 ; p<0,05$ para . tertius

*** $\mathrm{n}=1$ 
populações de $\mathrm{R}$ neglectus houve predomínio de ninfas (índice adulto/ninfa $=0,43$ ), enquanto as populações de P. tertius foram mais representadas por adultos ( índice adulto/ninfa $=1,26$ ). Já 0 padrão de distribuição por sexo mostrou ser semelhante, com maior número de machos nas populações. Um número relevante de ovos e ninfas de estágios 1 e 2 de R. neglectus foi coletado, indicando um evento reprodutivo no mês de fevereiro. No caso de P. tertius esse padrão ocorreu com menos intensidade.

Relações entre a infestação de triatomíneos, presença de ninhos, medidas das palmeiras e a paisagem. A diferença estatística na infestação em palmeiras com e sem ninhos foi muito significativa ( $X^{2}=48,8 ; p<0,01$ ) mostrando que a distribuição não é aleatória e fortemente influenciada pela presença de ninhos nas bainhas foliares das palmeiras. Houve uma correlação positiva entre o número de ninhos presentes nas bainhas foliares das palmeiras amostradas e 0 número total de $R$. neglectus $\left(r_{s}=0,66 ; p<0,01\right)$ e P. tertius $\left(r_{s}=0,62 ; p<0,01\right)$ coletados (Figura 1). 0 ninho do pássaro Furnariidae Phacellodomus ruber (graveteiro) ocorreu em $42 \%$ das palmeiras, apresentando as maiores abundâncias detriatomíneos. P. tertius só ocorreu em palmeiras que possuíam ninhos dessa espécie de ave, enquanto R. neglectus esteve associado a outros ninhos de aves (Gnorimopsar chopi - pássaro preto e Polyborus plancus caracará) e mamíferos ( Didelphis albiventris - gambá) nas bainhas foliares das palmeiras ( Tabela 3).

Considerando as 150 palmeiras medidas, foi observado um número médio $11,5 \pm 3,2$ folhas ( 3 a 20 folhas), altura média da estipe de $7,2 \pm 3.3 \mathrm{~m}(0,5$ a $17,0 \mathrm{~m})$ e diâmetro

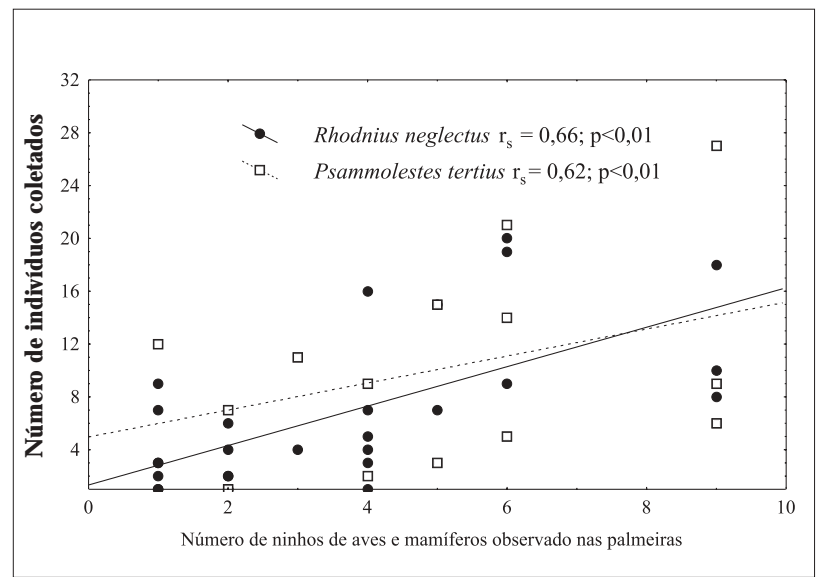

Figura 1 - Correlação de Spearman entre 0 número de ninhos presentes nas bainhas foliares de Mauritia flexuosa amostradas e 0 número total de Rhodnius neglectus e Psammolestes tertius coletados. médio de 7,2 $\pm 1,5 \mathrm{~m}$ ( 2 a 10,2m). As correlações entre as medidas das palmeiras e a abundância de $\mathrm{R}$. neglectus ( número de folhas: $r_{s}=-0,577$; altura: $r_{s}=0,125$; diâmetro da copa: $r_{s}=0,135$ ) e P. tertius (número de folhas: $r_{s}=-0,372$; altura: $r_{s}=0,407$; diâmetro da copa: $r_{s}=0,394$ ) não foram significativas para $\mathrm{p}<0,05$.

A abundância relativa de $\mathrm{R}$. neglectus e $\mathrm{P}$. tertius variou de acordo com a paisagem, densidade de palmeiras por hectare e número total de ninhos nas bainhas foliares ( Figura 2).

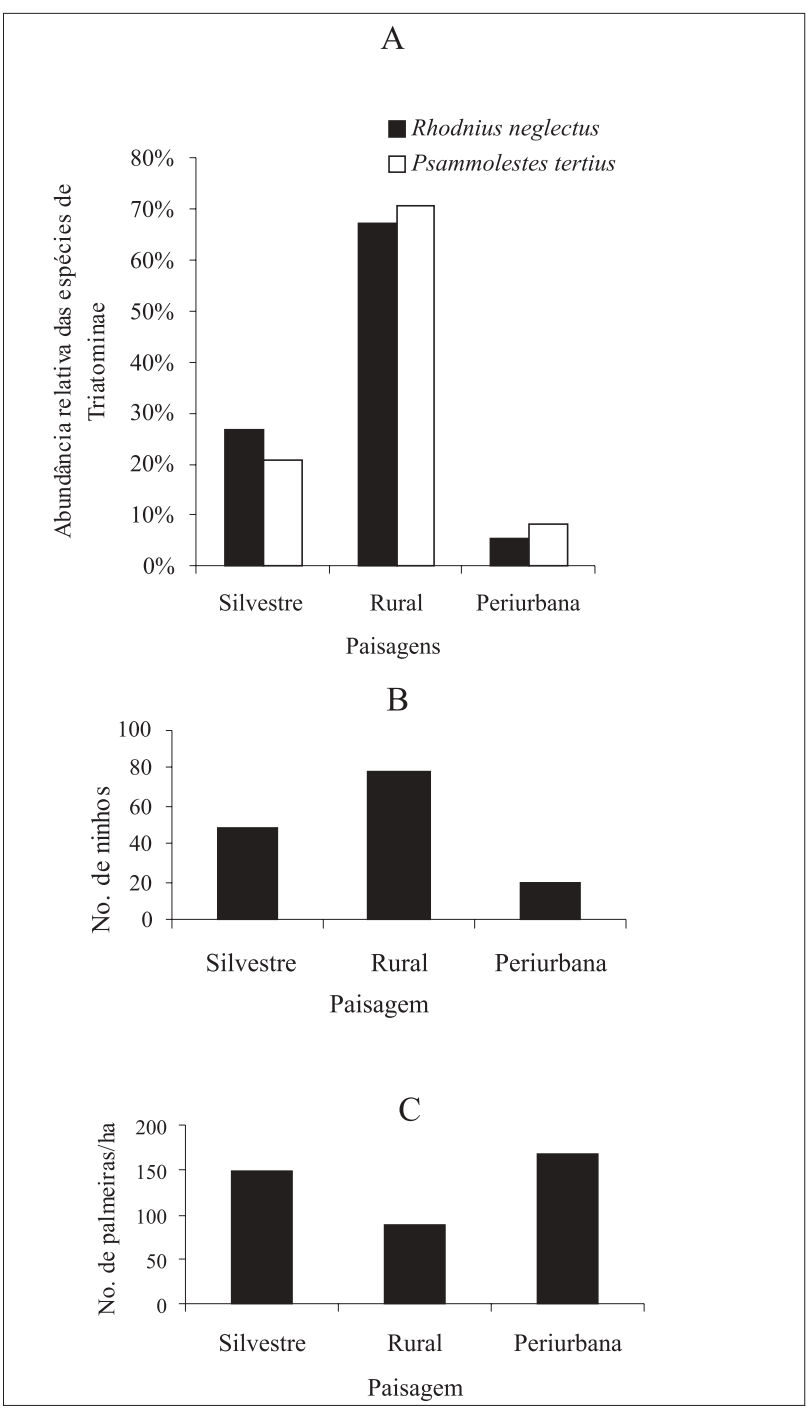

Figura 2-Relação entre a infestação de Rhodnius neglectus e Psammolestes tertius e as paisagens silvestre $(n=50)$, rural $(n=50)$ e periurbana $(n=50)$ no Distrito Federal, Brasil, 2003. A: abundância relativa das espécies nas paisagens; B: número de ninhos em cada paisagem; C: número de palmeiras por hectare em cada paisagem.

Tabela 3 - Número de insetos por palmeira e abundância relativa de Rhodnius neglectus e Psammolestes tertius em ninhos de aves e mamíferos presentes em Mauritia flexuosa no Distrito Federal, Brasil, 2003.

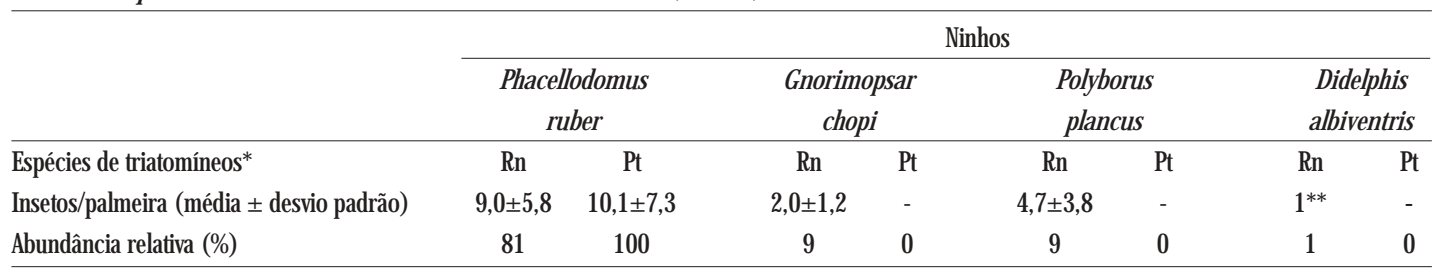

* Rn = Rhodnius neglectus, Pt = Psammolestes tertius, ${ }^{* *} \mathrm{n}=1$ 


\section{DISCUSSÃ0}

As espécies de triatomíneos encontradas foram $R$ neglectus e P. tertius cujas distribuições, freqüências e abundâncias na palmeira $\mathrm{M}$ flexuosa foram descritas pela primeira vez no $\mathrm{DF}$.

Em Uberaba (Minas Gerais), a freqüência de R. neglectus em 32 palmeiras da espécie M. flexuosa amostradas foi de 93,7\% com densidade de 15,6 indivíduos por palmeira4, em Mambaí (Goiás) essa freqüência foi de 65,6\% e densidade de 4,2 indivíduos por palmeira, pesquisando o mesmo número de palmeiras ${ }^{24}$ e em Frutal (Minas Gerais) a freqüência foi $69,2 \%$ e densidade de 9,5 indivíduos por palmeira, em treze palmeiras amostradas $^{13}$. No presente trabalho, a frequêencia variou de 12 a $40 \%$ e a densidade de 3,3 a 9,8 indivíduos por palmeira nas cinco áreas amostradas.

As variações de frequiência e abundância dos triatomíneos anteriormente citadas podem ser explicadas por vários fatores. No presente estudo, a amostragem foi padronizada para comparar as áreas e verificar fatores que influenciam a infestação. Porém, outros métodos de amostragem otimizando a busca dos triatomíneos ( selecionando palmeiras com ninhos, por exemplo) podem ter sido feitos pelos citados autores. Outro fator metodológico poderia ser a maior sensibilidade do método de dissecção após derrubada da palmeira em relação ao método de coleta manual ${ }^{19}$, mas para confirmar isso haveria a necessidade de comparar ambos os métodos em um mesmo local. Além dos fatores metodológicos, outros devem ser levados em consideração: densidade de palmeiras e grau de perturbação da vereda, abundância de fonte alimentar ou de predadores de triatomíneos (aranhas, pseudo-escorpiões, hemipteros predadores), diferenças climáticas locais entre áreas, além de diferenças na reprodução e dispersão das populações devido a época de amostragem.

Neste estudo, a composição etária das populações de R. neglectus investigadas mostrou predomínio de ninfas (índice adulto/ninfa: 0,43) , 0 que está de acordo com a estrutura etária geralmente observada em algumas populações de Rhodnius em palmeiras, com índice adulto ninfa inferior a 1, variando entre 0,36 e 0,9029. A coleta de ovos indica a existência de eventos reprodutivos no começo do ano (janeiro/fevereiro), durante a estação chuvosa. Resultados com populações de R. neglectus em palmeiras do gênero Acrocomia sugerem que há um grande crescimento das colônias nos meses mais quentes do ano (estação chuvosa) com alta produção de ovos, porém nos meses mais frios (estação seca) também foram capturados ovos, em menor quantidade ${ }^{11}$ indicando mais que um evento reprodutivo por ano.

Quanto a P. tertius, estudos anteriores indicaram frequiências superiores a 50\% em ninhos de Phacellodomus rufifrons (JoãoGraveto) em árvores do Cerrado ${ }^{2336}$. Neste trabalho, mostramos que P. tertius também forma colônias em ninhos de P. ruber (espécie de Furnariidae presente em Mauritia). A estrutura populacional de P. tertius, ao contrário de R. neglectus, mostrou um maior número de indivíduos adultos (índice adulto/ninfa: 1,23 ) , sendo poucos os indivíduos de estágios iniciais coletados. Padrão semelhante foi obtido para populações de P. tertius coletadas em ninhos de P. rufifrons, com índices adulto/ninfa de $1,10^{23}$ e $1,46^{36}$. Isso poderia ser explicado pela sobrevida longa dos adultos, alta mortalidade das ninfas e/ou por diferentes períodos de reprodução e dispersão. Apesar da espécie já ter sido listada ocorrendo em Mauritia ${ }^{413}$, esses estudos não mostraram a associação entre P. tertius e P. ruber. A freqüência e abundância de P. tertius em $\mathrm{M}$. flexuosa apresentaram valores menores, quando comparados com ninhos de P. rufifrons em árvores do Cerrado ${ }^{23}{ }^{36}$, sendo a presença desta espécie de triatomíneo na palmeira $\mathrm{M}$. flexuosa condicionada a presença do ninho de P. ruber. Isso sugere que os ninhos de P. ruber associados à palmeira $\mathrm{M}$. flexuosa, assim como os ninhos de P. rufifrons em árvores, seriam indicadores visuais da presença de populações de P. tertius no Cerrado.

A existência de ninhos nas bainhas foliares das palmeiras sugere ser uma das principais características que determinam uma maior abundância de R. neglectus e P. tertius em M. flexuosa. Os ninhos de P. ruber foram os que apresentaram maiores densidades de insetos. Essa espécie de pássaro é considerada sedentária, vivendo associada a campos úmidos, particularmente em palmeirais ocorrendo no Brasil (Bahia, Minas Gerais, Rio Grande do Sul), Bolívia, Paraguai e Argentina ${ }^{35}$. Os ninhos são construídos por gravetos entremeados de variados tamanhos ( 2 a $9 \mathrm{~cm}$ ) e possuem câmaras ou dormitórios no interior, formadas por fibras, folhas e outros materiais como papel e plástico, sendo este 0 local onde as populações de $R$ neglectus e $P$. tertius foram encontradas com maior frequiência. Normalmente, o mesmo local é usado por três ou mais anos consecutivos com uma nova câmara sendo sobreposta a antiga estrutura, fazendo com que o ninho alcance grandes dimensões? permitindo a colonização dessas espécies de triato míneos.

As diferentes densidades de triatomíneos encontradas em um ecótopo podem estar relacionadas com características estruturais do ecótopo que favoreçam a nidificação de vertebrados e consequentemente a colonização de triatomíneos. Entretanto, neste estudo, as características morfológicas de M. flexuosa (altura, diâmetro da copa e número de folhas) não foram correlacionadas com abundâncias de R. neglectus e P. tertius. Além disso, o número de ninhos encontrados nas palmeiras não obteve índices de correlação significativos com as características morfológicas das palmeiras, sugerindo que a nidificação das espécies de aves e mamíferos ocorre de forma aleatória.

Neste trabalho, observou-se que a freqüência $\mathrm{R}$. neglectus e P. tertius foi maior em áreas rurais, que possuíram maior número de ninhos nas bainhas foliares e menor densidade de palmeiras por hectare. A relação entre infestação de triatomíneos e paisagem tem sido descrita em estudos envolvendo $\mathrm{R}$. pallescens em palmeiras da espécie Attalea butyracea. № Panamá, em paisagens periurbanas, com menor densidade de palmeiras, há um maior número de insetos por palmeira, diferindo muito das áreas de floresta com alta densidade de palmeiras e baixa densidade de insetos por palmeira ${ }^{22}$. Na Colômbia, um padrão semelhante foi observado com R. pallescens e A. butyracea ${ }^{29}$. Segundo Romaña et $\mathrm{al}^{31}$, 0 manejo da paisagem pelo homem com desmatamento, pasto e urbanização poderia alterar as dinâmicas populacionais das palmeiras e, em consequiência, as densidades de populações de Rhodnius, influenciando a ecoepidemiologia da tripanossomíase Americana. 
Ainvasão de espécies do gênero Rhodnius em novos habitats, inclusive habitações humanas, poderia ocorrer devido à carência nutricional nos ecótopos naturais ou devido a estratégias de dispersão, sendo a presença de fontes luminosas artificiais e a sazonalidade ${ }^{26}$ fatores que poderiam influenciar esse processo. Desequilíbrios ecológicos decorrentes de desmatamento poderiam diminuir a oferta de fontes alimentares nesses ecótopos favorecendo a entrada dos vetores no ambiente domiciliar, principalmente à noite, atraídos pela luz.

Rhodnius neglectus é um bom exemplo de espécie silvestre que invade ocasionalmente ecótopos artificiais, formando colônias e podendo transmitir T. cruzi, diferentemente de P. tertius, espécie estritamente silvestre ${ }^{36}$. A presença de R. neglectus nas habitações humanas e anexos vem sendo assinalada há muito tempo em São Paulo, Goiás e Minas Gerais, onde podem formar pequenas colônias, com taxa média de infecção de 3,4\%3. Experimentalmente, a capacidade de dispersão e domiciliação de $\mathrm{R}$. neglectus foi demonstrada com a colonização espontânea de galinheiros artificiais no perimomicílio, sendo a proximidade das palmeiras um fator importante para ocorrer essa dispersã $0^{13}{ }^{14}$. Em Goiás, durante 1975 e 1982, 41\% dos municípios reportaram a infestação de R. neglectus no domicílio, sendo que $43 \%$ das capturas foram feitas no intradomicílio ${ }^{15}$. Nos distritos de Formosa, Jataí, Morrinhos e Ceres, em Goiás, R. neglectus foi a segunda espécie com maior incidência no ambiente domiciliar ${ }^{33}$. No Paraná, esta espécie foi capturada em casas abandonadas na área rural ${ }^{18}$. Os levantamentos entomológicos feitos pela FUNASA/Secretaria de Vigilância Ambiental do D.F., mostram poucos R. neglectus coletados no ambiente domiciliar nos últimos anos. Apesar deste estudo não ter mostrado a infecção de R. neglectus por T. cruzi ou T. rangeli, a transmissão enzoótica desses parasitas tem sido evidenciada no DFenvolvendo R. neglectus ${ }^{19}$. Portanto, as palmeiras da espécie $\mathrm{M}$. flexuosa poderiam ser potenciais indicadores ecológicos da presença de focos de transmissão de T. cruzi no DF, porém o risco de infecção humana deve ser cuidadosamente mensurado e avaliado.

\section{AGRADECIMENTOS}

Os autores agradecem a colaboração do Fábio e sua família no trabalho de campo e aos colegas Liléia Diotaiuti, Guy Mejia e Michael Miles por revisar versões preliminares do trabalho.

\section{REFERÊNCIAS BIBLIOGRÁFICAS}

1. Assis AL. Vigilância epidemiológica da doença de Chagas no Distrito Federal. Monografia de especialização em Saúde Coletiva, Faculdade de Ciências da Saúde, Universidade de Brasília, Brasília, DF, 1999.

2. Barrett TV. Current research on Amazonian Triatominae. Memórias do Insituto Oswaldo Cruz 83 ( suppl I) : 441-447, 1988.

3. Barretto MP. Epidemiologia. In: Brener Z Andrade ZA(ed) Trypanosoma cruzi e Doença de Chagas, Guanabara Koogan Ed, Rio de Janeiro, p. 89-291, 1979.

4. Barretto MP, Albuquerque DRR, Funayama GK. Estudos sobre reservatórios e vectores silvestres do Trypanosoma cruzi. XXXVI: Investigação sobre triatomíneos de palmeiras no município de Uberaba, MG, Brasil. Revista Brasileira de Biologia 29:577-588, 1969.

5. Bento DNC, Branco AZC, Freitas MR, Pinto AS. Epidemiologic studies of Chagas' disease in the urban zone of Teresinha, state of Piaui, northeastern Brazil. Revista da Sociedade Brasileira de Medicina Tropical 17: 199-203, 1984.

6. Carcavallo RU, Rodríguez MEF, Salvatella R, Curto de Casas SI, Sherlock I, Galvão C, Rocha DS, Galíndez Girón I, Arocha MAO, Martinez A, Da Rosa JA, Canale DM, Farr TH, Barata JMS. Habitats and related fauna. In: Carcavallo RU, Galíndez Girón I, Jurberg J, Lent H (eds) Atlas of Chagas Disease Vectors in Americas, vol II, Editora FIOCRUZ, Rio de Janeiro, p. 561-600, 1998.

7. Carrara LA, Rodrigues M. Breeding biology of the rufous-fronted thornbird Phacellodomus rufifrons, a Neotropical ovenbird. International Journal of Ornithology 4: 209-217, 2001.

8. Cuba-Cuba CA. Estudo de uma cepa peruana de Trypanosoma rangeli IV. Observações sobre a evolução e morfogênese do T. rangeli na hemocele e glândulas salivares de Rhodnius ecuadoriensis. Revista do Instituto de Medicina Tropical de São Paulo 17: 284-297, 1975.

9. Cuba-Cuba CA, Alvarenga N, Barretto AC, Marsden PD, Macedo V, Gama MP. Dipetalogaster maximus ( Hemiptera, Triatominae) for xenodiagnosis of patients with serologically detectable T. cruzi infections. Transactions of the Royal Society of Tropical Medicine and Hygiene 73: 524-527, 1979.

10. D' Alessandro A, Barretto P, Saravia N, Barretto M. Epidemiology of Trypanosoma cruzi in the oriental plains of Colombia. American Journal of Tropical Medicine and Hygiene 33: 1084-1095, 1984.

11. Diotaiuti L, Dias JCP. Ocorrência e biologia de Rhodnius neglectus, Lent, 1954 em macaubeiras da periferia de Belo Horizonte, Minas Gerais. Memórias do Instituto Oswaldo Cruz 79:293-301, 1984.

12. Dujardin JP, Garcia-Zapata MT, Juberg L, Roelants P, Cardozo L, Panzera F, Dias JCP, Schofield CJ. Which species of Rhodnius is invading houses in Brazil? Transactions of the Royal Society of Tropical Medicine and Hygiene 85: 679-680, 1991.

13. Forattini OP, Ferreira OA, Rabello EX, Barata JMS, Santos JLF. Aspectos ecológicos da Tripanossomíase Americana XVII Desenvolvimento da domiciliação triatomínea regional em centro de endemismo de Triatoma sordida. Revista de Saúde publica de São Paulo 17: 159-199, 1983.

14. Forattini OP, Rocha e Silva EO, Ferreira OA, Rabello EX, Patton DGB. Aspectos ecológicos da Tripanossomíase Americana III Dispersão local de triatomíneos, com especial referência ao Triatoma sordida. Revista de Saúde publica de São Paulo 5: 193-205, 1971.

15. Garcia-Zapata MT, Marsden PD. Control of the transmission of Chagas' disease in Mambai, Goiás, Brazil (1980-1988). American Journal of Tropical Medicine and Hygiene 46: 440-443, 1992.

16. Gaunt MF, Miles MA. The ecotopes and evolution of triatomine bugs (Triatominae) and their associated trypanosomes. Memórias do Instituto Oswaldo Cruz 95: 557-565, 2000.

17. Greig-Smith P. Quantitative plant ecology. University of California Press, Berkeley, California, USA. p.163-164, 1983.

18. Guilherme AL, Pavanelli GC, Silva SV, Costa AL, de Araújo SM. Secondary triatomine species in dwellings and other nearby structures in municipalities under epidemiological surveillance in state of Parana, Brasil. Revista Panamericana de Salud Publica 9: 385-392, 2001.

19. Gurgel-Goncalves R, Palma ART, Menezes MNA, Leite RN, Cuba Cuba CA. Sampling Rhodnius neglectus (Triatominae) in Mauritia flexuosa palm trees (Arecaceae): a field study in the Brazilian Savanna. Medical and Veterinary Entomology 17: 347-349, 2003.

20. Lent H, Wygodzinsky P. Revision of the triatominae (Hemiptera, Reduviidae), and their significance as vectors of Chagas Disease. Bulletin American Museum of Natural History 163: 520-529, 1979.

21. Martins RC. Arecaceae ( Palmae) no Distrito Federal (Brasil) . Acta Botânica Brasileira 15: 288, 2001

22. Mejì GD. Distribuicion espacial de Rhodnius pallescens Barber, 1932 en la Provincia de Panama, Republica de Panama. Monografia de la carrera de Biologia, Universidad del Atlantico, Barranquilla, Colombia, 2003. 
23. Mello DA. Aspectos do ciclo silvestre do Trypanosoma cruzi em regiões do cerrado (Município de Formosa, Goiás). Memórias do Instituto Oswaldo Cruz 76: 227-246, 1981.

24. Mello DA. Roedores, marsupiais e triatomíneos silvestres capturados no município de Mambaí-Goiás. Revista de Saúde pública 16: 282-291, 1982.

25. Miles MA, Arias JR, de Souza AA. Chagas Disease in Amazon basin: V Periurban palms and habitats of Rhodnius robustus and Rhodnius pictipes - triatomine vectors of Chagas Disease. Memórias do Instituto Oswaldo Cruz 78: 391-398, 1983

26. Naiff MF, Naiff RD, Barrett TV. Vetores selváticos de doença de Chagas na área urbana de Manaus (AM): atividade de vôo nas estações secas e chuvosas. Revista da Sociedade Brasileira de Medicina Tropical 31:103105, 1998.

27. Noireau F, Abad-Franch F, Valente SAS, Dias-Lima A, Lopes CM, Cunha V, Valente VC, Palomeque FS, Carvalho-Pinto CJ, Sherlock I, Aguilar M, Steindel M, Grisard EC, Juberg J. Trapping Triatominae in Sylvatic Habitats. Memórias do Instituto Oswaldo Cruz 97: 61-63, 2002.

28. Pinto AS, Bento DNC. The palm tree Copernicia cerifera (Carnaúba) as an ecotope of Rhodnius nasutus in rural areas of the state of Piaui northeastern Brazil. Revista da Sociedade Brasileira de Medicina Tropical 19: 243-245, 1986.

29. Pizarro JC, Romaña CA. Variación estacional de una población silvestre de Rhodnius pallescens Barber, 1932 (Heteroptera: Triatominae) en la costa caribe Colombiana. Bulletin de l'Institut français d' études andines 27: 309-325, 1998.

30. Ribeiro JF, Walter BMT. Fitofisionomias do bioma Cerrado. In: Sano SM, Almeida SP (eds) Cerrado Ambiente e Flora, Embrapa, Planaltina, Brasil, 1998.

31. Romaña CA, Brunstein D, Collin-Delavaud A, Souza 0, Ortega-Barria E. Public policies of development in Latin America and Chagas Disease. Lancet 362: 579, 2003
32. Romaña CA, Pizarro JCN, Rodas E, Guilbert E. Palm trees as ecological indicator of risk areas for Chagas disease. Transactions of the Royal Society of Tropical Medicine and Hygiene 93: 594-595, 1999.

33. Santos AH, Isac E, Silva JL, Camargo MF, Oliveira AWS, Silva EAO, Abrão N. Índices de infestação domiciliar e infecção natural pelo Trypanosoma cruzi das espécies de triatomíneos capturadas no estado de Goiás, no período 1994-1998. In: Resumos do XIV Congresso Brasileiro de Parasitologia, Poços de Caldas p. 150, 1999.

34. Schofield CJ, Dujardin JP. Theories on the Evolution of Rhodnius. Actualidades Biológicas (Medellín) 21: 183-197, 1999.

35. Sick H. Ornitologia Brasileira. Editora Nova Fronteira, Rio de Janeiro, 1997.

36. Silva IG, Lustosa ES. Biologia de Psammolestes tertius Lent \& Jurgberg, 1965 (Hemiptera, Reduviidae). Revista de Patologia Tropical 22: 29-42, 1993.

37. Silveira AC, Feitosa VR, Borges R. Distribuição de triatomíneos capturados no ambiente domiciliar, no período de 1975/83, Brasil. Revista Brasileira de Malariologia e Doenças Tropicais 39: 15-312, 1984.

38. Silveira AC, Vinhaes M. Atlas da epidemiologia e do controle de doenças endêmicas no Brasil. Revista da Sociedade Brasileira de Medicina Tropical 31 ( suppl II) :15-60, 1998.

39. Teixeira ARL, Monteiro PS, Rebelo JM, Arganaraz ER, Vieira D, LauriaPires L, Nascimento R, Venexat CA, Silva AR, Ault SK, Costa JM. Emerging Chagas disease: trophic network and cycle of transmission of Trypanosoma cruzi from palm trees in the Amazon. Emerging Infectious Diseases 7: 100-112, 2001

40. Valente VC, Valente SAS, Rodrigues CA, Souza GCR, Noireau F, Lopes CM, Cunha V. Estudo preliminar da eficiência de captura de triatomíneos silvestres utilizando armadilhas com fita adesiva em Bragança Pará. Revista da Sociedade Brasileira de Medicina Tropical 33 ( suppl II): 94-95, 2000.

41. Zar JH. Biostatistical analysis. Prentice Hall International, INC Upper Saddle River, New Jersey, 1996. 